Clasificación del hábitat potencial del tapir centroamericano

(Tapirus bairdii Gill, 1865) para su conservación en Guatemala

\title{
Classification of the potential habitat of the Central American tapir (Tapirus bairdii Gill, 1865) for its conservation in Guatemala
}

\author{
Manolo García ${ }^{1 *}$ y Raquel Leonardo²
}

\author{
${ }^{1}$ Centro de Estudios Conservacionistas de la Universidad de San Carlos de Guatemala. Avenida Reforma 0-63 zona 10, Ciudad de \\ Guatemala 01010, Guatemala. E-mail: garcia.manolo@usac.edu.gt (MG). \\ ${ }^{2}$ Unidad de Investigación, Fundación Defensores de la Naturaleza. 4 Avenida 23-01, Zona 14, Ciudad de Guatemala 01014, \\ Guatemala. E-mail: raque.leonardo@gmail.com (RL) \\ ${ }^{*}$ Corresponding author
}

Tapir distribution in Guatemala has dropped dramatically over the past century, causing populations to be restricted to protected areas of the northeastern region of the country. This study seeks to determine the conservation status of the species habitat in the country based on its presence, the importance of forest remnants in system connectivity and coverage of formal mechanisms of protection. The Maxent distribution modeling was performed using 62 records of presence 10 climatic variables and terrain elevation. The resulting map was reclassified into a binary map (cutoff 0.3 ) and using the Morphological Spatial Pattern Analysis tool of GUIDOS program, a map in which the 12 larger forest remnants were selected was obtained. To these remnants the Integral Connectivity Index (dIIC) with Sensinode CONEFOR program was calculated. From the above, classifications of forest remnants were established to determine the conservation status of the species habitat. The potential distribution model had an area under the curve (AUC) of 0.815 and from the binary map it was estimated that the potential habitat of the tapir in 2012 was $26,095.82 \mathrm{~km}^{2}$. The Morphological Spatial Pattern Analysis showed that the tapir habitat is mainly composed of core area (43\%). The connectivity analysis indicated that the forest remnants of the Maya Biosphere Reserve (Northeast and West) and the Sierra de las Minas Biosphere Reserve have a dIIC of 33.505662, 0.51311405 and 0.38876015, respectively. The remnants of larger extension and higher dIIC correspond to the northeast and west section of the Maya Biosphere Reserve and Sierra de las Minas Biosphere Reserve. Compared with previous studies, there is a tendency in reducing coverage and degradation (fragmentation) tapir habitat, highlighting the vulnerability of the species populations in the country.

La distribución del tapir en Guatemala se ha reducido drásticamente durante el último siglo, provocando que sus poblaciones estén restringidas a las áreas protegidas de la región nororiente del país. En este estudio se determinó el estado de conservación del hábitat para la especie en el país basado en su presencia, la importancia de los remanentes boscosos en la conectividad del sistema y la cobertura de mecanismos formales de protección. Se realizó el modelado de distribución con Maxent, utilizando 62 registros de presencia, 10 variables climáticas y la elevación. El mapa resultante se reclasificó en un mapa binario (umbral de corte 0.3) y utilizando la herramienta Morphological Spatial Pattern Analysis del Programa GUIDOS, se obtuvo un mapa en el que se seleccionaron los 12 remanentes boscosos de mayor superficie. A estos remanentes se les calculó el Índice Integral de Conectividad (dIIC) con el programa CONEFOR Sensinode. A partir de lo anterior se establecieron clasificaciones de los remanentes boscosos para establecer el estado de conservación de los mismos. El modelo de distribución potencial tuvo un área bajo la curva (AUC) de 0.815 y a partir del mapa binario se estimó que el hábitat potencial para el tapir en el 2012 era de 26,095.82 km². El Análisis Morfológico de los Patrones Espaciales mostró que el hábitat del tapir está compuesto principalmente de área de interior (43\%). El análisis de conectividad indicó que los remanentes boscosos de la Reserva de Biosfera Maya (Noreste y Oeste) y la Reserva de Biosfera Sierra de las Minas tienen un dIIC de 33.505662, 0.51311405 y 0.38876015 respectivamente. Los remanentes de mayor superficie e Índice Integral de Conectividad corresponden a la sección noreste y oeste de la Reserva de Biosfera Maya y a la Reserva de Biosfera Sierra de las Minas. En comparación con estudios previos, existe una tendencia en la reducción de la cobertura y fragmentación del hábitat del tapir, evidenciando la vulnerabilidad de las poblaciones silvestres de la especie en el país.

Key words: Potential distribution; Morphological Spatial Pattern Analysis; Integral Connectivity Index (dIIC). 


\section{Introducción}

El Tapir centroamericano (Tapirus bairdii; Perissodactyla: Tapiridae), también conocido como tixl (en idioma Q'eqchi'), es el mamífero terrestre de mayor talla de Guatemala y de la región Mesoamericana. Su distribución regional comprende desde el sureste de México hasta el este de Ecuador y Colombia (Matola et al. 1997; Castellanos et al. 2008; Naranjo 2009; Schank et al. 2015). Se le puede encontrar desde el nivel del mar hasta los $3620 \mathrm{~m}$ de altura (Naranjo y Vaughan 2000), habitando en diversos ambientes tropicales y subtropicales, entre ellos bosques tropicales, manglares, bosques mesófilos, encinares y páramos (March 1994; Naranjo 2001).

De acuerdo a la Unión Internacional para la Conservación de la Naturaleza (IUCN) el tapir se encuentra en la lista roja de especies amenazadas debido a la reducción de sus poblaciones (Castellanos et al. 2008). A nivel regional y local las principales amenazas son la pérdida y degradación del hábitat, y en menor medida la cacería (Matola et al. 1997; Castellanos et al. 2008). Debido a los requerimientos específicos de calidad hábitat, la presencia del tapir constituye un indicador del buen estado de conservación de las áreas donde aún está presente (Zapata y Dyer 2003; Botello et al. 2014).

Las primeras descripciones de la distribución de la especie para el país corresponden a la segunda mitad del siglo XIX, y abarcan la costa atlántica y pacífica guatemalteca, incluyendo las faldas de la cadena volcánica de la Sierra Madre, en el descenso del altiplano hacia la planicie costera del Pacífico, con elevaciones de 300 a 1,400 m (Alston 1918; Ximenez 1967; www.insivumeh.gob. gt). El conocimiento sobre la distribución del tapir se enriquece a mediados del siglo XX, cuando se reportó que el área de distribución también comprendía los departamentos de Petén, Quiché y Alta Verapaz (Saunders et al. 1950; Ibarra 1959). A finales de la década de 1990, Matola et al. (1997) reportaron que el tapir estaba presente solamente en cuatro departamentos, siendo éstos Quiché, Alta Verapaz, Izabal y Petén, evidenciando de esta manera su extinción en la región sur del territorio nacional. A inicios del presente siglo se iniciaron los estudios específicos sobre el tapir en el país (al igual que otros estudios de fauna), por medio de los cuales fue posible verificar (a través de huellas, excretas, fototrampeo y material de colecciones científicas) que su distribución actual está restringida a los departamentos de Alta Verapaz, Baja Verapaz, Quiché, El Progreso, Izabal y Petén (Kawanishi 1995); Baur 1999; Bestelmeyer y Alonso 2000; Novack 2003; García y Radachowsky 2004; Phillips y Dudik, 2008; Morales et al. 2005; Ruíz y García 2005; Ruíz y Rodríguez 2006; García 2006; Moreira et al. 2007; García et al. 2008a, 2008b, 2009, 2010a, 2010b; Gómez 2011).

Siendo las principales amenazas para el tapir la cacería y la pérdida de hábitat, se puede deducir que la extinción local de la especie está asociada con el cambio del uso del suelo y la consecuente fragmentación del hábitat (Castellanos et al. 2008). En este escenario, los remanentes de hábitat y el patrón espacial de los mismos, son elementos de relevancia para la conservación de la especie en el país. En el presente estudio se busca desarrollar una clasificación del hábitat del tapir en el país que pueda ser aplicada en la planificación y ordenamiento del territorio. La clasificación propuesta se basa en tres aspectos: presencia de la especie en la última década (2005-2015), la importancia de cada remanente boscoso para la conectividad del sistema y la extensión bajo mecanismos de protección formal dentro de cada remanente boscoso.

\section{Materiales y métodos}

Área de estudio. El área de estudio comprendió toda la superficie del territorio guatemalteco, entre las latitudes $13.7^{\circ}$ y $17.8^{\circ} \mathrm{N}$ y longitudes $-88.2^{\circ}$ y $-92.2^{\circ} \mathrm{O}$, abarcando una extensión continental de $108,889 \mathrm{~km}^{2}$. Se recopilaron registros de presencia de tapir a través de visitas a 26 de las 34 áreas protegidas ubicadas dentro del ámbito de distribución reportado por Matola et al. (1997), 
durante las cuales se recorrieron los sitios sugeridos previamente por los guías, pobladores, registros históricos y conversaciones con técnicos, guarda recursos y administradores de las áreas protegidas. Durante las visitas de campo se realizaron caminatas para buscary referenciar los rastros que evidenciaban la presencia de la especie (huellas, excretas, comederos, echaderos y caminos). La fase de campo fue realizada entre los años 2007 y 2009 en áreas del Sistema Guatemalteco de Áreas Protegidas (SIGAP). Estos datos fueron complementados con registros de presencia de tapir en el país, correspondientes a registros actuales e históricos de huellas, excretas, comederos, echaderos y fototrampeo, generados a partir de investigaciones científicas (Ruíz y García 2005; Ruiz y Rodríguez 2006; García et al. 2008b; 2009b), bases de datos de los programas de monitoreo biológico de instituciones locales (Fundación Mario Dary -FUNDARY- datos del 2005-2007; Centro de Estudios Conservacionistas de la Universidad de San Carlos de Guatemala (CECON) datos del 2014-2015; Fundación Defensores de la Naturaleza (FDN) datos del 2014) y revisión bibliográfica. Todos estos datos fueron compilados en una base de datos digital.

Modelado de la distribución potencial. El modelado de distribución potencial del tapir se realizó utilizando el algoritmo Maxent Ver. 3.3.3 k (Phillips y Dudik, 2008). Para ello se utilizaron 10 variables climáticas (BIO1, $\mathrm{BIO} 4, \mathrm{BIO} 5, \mathrm{BIO} 6, \mathrm{BIO} 12, \mathrm{BIO} 15, \mathrm{BIO} 16, \mathrm{BIO} 17, \mathrm{BIO} 18$ y $\mathrm{BIO} 19)$ y la elevación (ALT), en formato raster con resolución de $1 \mathrm{~km}^{2}$, provenientes de la base de datos versión 1.4 de WorldClim (Hijmans et al. 2005), siguiendo a Schank et al. (2015).

Dado que se cuenta únicamente con tres registros para la vertiente del Pacífico, donde de acuerdo con Alston (1918) la especie aún era abundante a principios del siglo pasado, se asume que existe un sesgo en los registros de presencia actual con respecto a su distribución potencial, ya que no se cuenta con registros de localidades que fueron adecuadas para la especie pero que en la actualidad han sido deforestadas (GIMBOT, 2014). Debido a lo anterior con el fin de contar con una distribución más uniforme de los registros de presencia para reducir este sesgo y el efecto de un mayor muestreo adentro de áreas protegidas, utilizando ArcMap ver. 10 (ESRI, Redlands, CA, EE. UU.) se generó una cuadrícula de $10 \times 10 \mathrm{~km}^{2}$ que se sobrepuso a la capa de registros de presencia existentes y se procedió a seleccionar únicamente un registro para cada celda, identificando de manera visual el registro (de la base de datos compilada) ubicado más cerca del centro de la celda. Por último se seleccionaron las opciones de curvas de respuesta, Jacknife y los ajustes preseleccionados por Maxent (default settings).

Análisis morfológico de los patrones espaciales. El mapa de distribución potencial generado con Maxent se reclasificó en ArcMap 10 a partir del umbral de corte de 0.3, que se estableció con base en las categorías utilizadas por Mendoza et al. (2013) y Schank et al. (2015) (incluyendo 3-5 low suitability, $5-7$ very suitable $y>7$ high suitability). El mapa binario resultante fue transformado a formato vectorial para realizar una extracción del Mapa de Cobertura Forestal por Tipo y Subtipo de Bosques en Guatemala (GIMBOT, 2014), y así obtener un mapa de los remanentes de hábitat para el tapir para el año 2012 con una resolución de $5 \times 5 \mathrm{~m}$. El resultado fue transformado de nuevo a un mapa binario formato raster con una resolución de 250 × $250 \mathrm{~m}$.

Con el fin de determinar el patrón morfológico espacial del hábitat potencial remanente, el mapa binario de presencia-ausencia correspondiente a las zonas con mayor probabilidad de distribución de la especie se procesó con la herramienta Morphological Spatial Pattern Analysis (MSPA) del Programa GUIDOS ver. 1.3, la cual utiliza una secuencia de operadores matemáticos para clasificar los objetos de una imagen en clases de acuerdo a su geometría, arreglo espacial y conectividad estructural (Vogt et al. 2007; Soille y Vogt 2009). El resultado de este procesamiento proporcionó al mapa del hábitat potencial valores correspondientes a las siete clases del MSPA: área de interior (core area), relicto (islet), perforación (perforation), borde (edge), puente (bridge), lazo (loop) y rama (branch) (Vogt et al. 2007; Correa y Mendoza 2013). A partir de este mapa se generó un mapa con los principales remanentes de hábitat del tapir de 
acuerdo a su patrón morfológico espacial, incluyendo solamente las áreas interiores, sus bordes y las perforaciones.

Conectividad de los remanentes de hábitat. La capa de remanentes de hábitat resultante se transformó en formato vectorial para ser procesada con el programa CONEFOR Sensinode ver. 2.5.8 (Pascual-Hortal y Saura 2006; Saura y Pascual-Hortal 2007). A través de este programa se evaluó la conectividad funcional potencial para el tapir, estimando el diferencial del Índice Integral de Conectividad (dIIC) a cada uno de los remanentes de hábitat, el cual se calcula a partir de su conectividad interna, su contribución con el flujo y su contribución como conector (stepping stones). La distancia umbral de dispersión utilizada entre dos remanentes de hábitat para el tapir fue de 1,500m, con base a los datos de Medici (2010) para el tapir de tierras bajas Tapirus terrestris en Brasil. Este procedimiento permitió identificar los remanentes de hábitat de mayor importancia de acuerdo a su aporte a la conectividad de todo el sistema.

Clasificación de principales remanentes de hábitat potencial. Con base en la información generada a partir del modelado de distribución potencial, el análisis morfológico de los patrones espaciales, el Índice Integral de Conectividad (dIIC) y el mapa de distribución del tapir a nivel regional (Schank et al.2015), se desarrolló una propuesta de clasificación para los principales remanentes de hábitat potencial del tapir en el país. Tomando estos tres aspectos como base, se generó un cuadro de ponderación para los remanentes de hábitat potencial (modificado de García et al. 2008b, 2009). Se evaluaron los 25 remanentes con la mayor extensión con el fin de limitar el análisis a un número determinado. Los remanentes con afinidad espacial y administrativa, pertenecientes a una misma área protegida o región específica fueron reclasificados en complejos.

En la clasificación desarrollada se proponen cinco categorías (A1, A2, A3, B1 y B2) para la clasificación del hábitat potencial del tapir, las cuales representan la relevancia de los principales remanentes para la conservación de la especie en el país a partir de la presencia actual de la especie, el Índice Integral de Conectividad y la presencia de mecanismos formales de protección de la cobertura forestal y la diversidad biológica (áreas protegidas). Los remanentes tipo A corresponden a localidades donde la especie está presente en la actualidad y las B donde ya no está presente o no se tienen reportes recientes. Los remanentes A1 corresponden a zonas de gran extensión territorial, donde la conectividad es alta y existen mecanismos formales de protección (áreas protegidas oficialmente declaradas). Los remanentes tipo A2 son aquellos que albergan poblaciones de tapires pero que su conectividad es media. En los remanentes A3, la especie está presente y poseen una baja conectividad. En los remanentes tipo B1 la conectividad es baja y existen mecanismos formales de protección y los remanentes B2 se diferencian de los primeros por no presentar mecanismos formales de protección.

\section{Resultados}

Modelado de la distribución potencial. La base de datos de registros de presencia de tapir que se generó contiene un total de 206 registros con referencia espacial, de los cuales 148 corresponden a la verificación en campo durante el período 2007-2009 en 15 áreas protegidas del país (Biotopo Protegido Naachtún-Dos Lagunas, Biotopo Protegido San Miguel La Palotada-El Zotz, Concesión Forestal Carmelita (ZUM), Concesión Forestal Uaxactún (ZUM), Parque Nacional Laguna del Tigre, Parque Nacional Laguna Lachuá, Parque Nacional Mirador-Río Azul, Parque Nacional Sierra del Lacandón, Parque Nacional Tikal, Parque Nacional Yaxhá-Nakum-Naranjo, Refugio de Vida Silvestre Machaquilá, Reserva Biológica San Román, Reserva Comunitaria Indígena Bioitzá (ZUM), Reserva de Biosfera Montañas Mayas Chiquibul y Reserva de Biosfera Sierra de las Minas), 15 corresponden al programa de monitoreo biológico desarrollado por instituciones locales, 28 a comunicaciones personales y 15 a registros bibliográficos. Para el modelo se utilizaron 62 registros de presencia 
seleccionados con base a la metodología para obtener una distribución uniforme de los registros. El modelo de distribución potencial dio como resultado un área bajo la curva (AUC) de 0.815 . Las variables con los mayores porcentajes de contribución fueron $\mathrm{BIO} 17$ = Precipitación del trimestre más seco (38.8\%), $\mathrm{BIO} 4=$ Temperatura estacional (desviación estándar * 100) (27.9\%) y BIO15 = Coeficiente de variación de las precipitaciones estacionales (8.4\%).

Los valores de Maxent se presentan en un rango que va de 0 a 1, donde los valores más cercanos a 1 indican mayor probabilidad de ocurrencia del tapir, debido a una mejor condición del hábitat. De acuerdo a lo anterior en el modelo se observa que la mayor probabilidad de presencia de la especie está focalizada en la región nororiente del país (Petén, Izabal, Alta Verapaz, Baja Verapaz y una región de Quiché), siendo aún mayor en el noreste y oeste de esta región. Además, sobresalen pequeñas zonas con alta probabilidad de distribución ubicadas a lo largo de la cadena volcánica, al sur del territorio nacional (Figura 1). El resto del territorio presenta una baja probabilidad de distribución.

Análisis morfológico de los patrones espaciales. El mapa binario que se generó indica que la extensión total de hábitat potencial para el tapir abarca alrededor de $58,456.87 \mathrm{~km}^{2}$, es decir el $53.68 \%$ del territorio nacional, sin embargo, al eliminar las áreas donde no existe cobertura forestal, la extensión del hábitat potencial se reduce a 26,095.82 km², equivalente al $44.54 \%$ de su extensión potencial (GIMBOT, 2014).

La clasificación morfológica de los patrones espaciales permitió conocer mejor

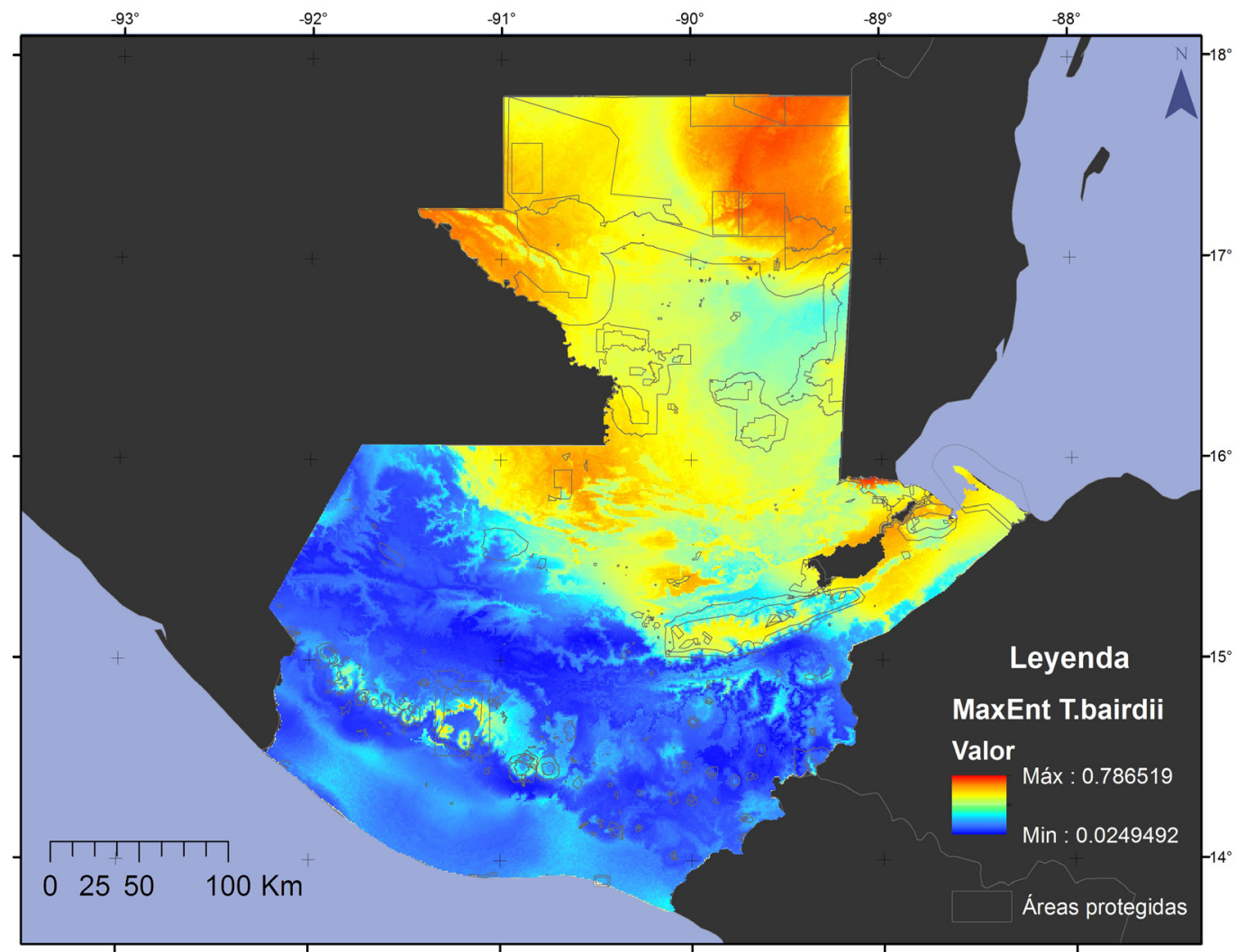

Figura 1. Modelo de distribución potencial de Tapirus bairdii en Guatemala generado con Maxent. Las áreas de color rojo muestran mayor probabilidad de ocurrencia de la especie y las áreas en color azul muestran la menor probabilidad de ocurrencia. 
la dinámica de los fragmentos de hábitat. En la Tabla 1 y Figura 2 se presentan los resultados de las siete clasificaciones de la cobertura, correspondientes al mapa binario de presencia-ausencia.

La herramienta MSPA estimó que el mayor porcentaje del hábitat del tapir al 2012 corresponde a área de interior ( $43 \%$ ), seguido de los elementos conectores, que incluye las clasificaciones lazo, puente y rama, que en su conjunto representan el $32 \%$. Sin embargo, es importante resaltar que la mayoría de los elementos fueron clasificados como relictos (remanentes de bosque aislados) y borde (4,620 y 1,712 respectivamente) a pesar de no presentar porcentajes elevados (14\% y $10 \%$ respectivamente).

Se seleccionaron los 25 remanentes de mayor superficie que están ubicados en el ámbito de distribución de la especie, los cuales fueron organizados en 12 unidades espaciales (remanentes o complejos de remanentes) que se describen en la Tabla 2.

Conectividad de los remanentes de hábitat potencial. El resultado del análisis de conectividad muestra que el remanente con el mayor dIIC es el Noreste de la Reserva de Biosfera Maya (RBM-NE; 33.505662). El resto de remanentes presentan una baja conectividad, a excepción de los remanentes Sierra de las Minas y el Oeste de la Reserva de Biosfera Maya (RBM-O) que son de mayor tamaño (0.51311405 y 0.38876015 respectivamente). El resto de remanentes presenta un Índice Integral de Conectividad menor a 0.1 (Tabla 3 y Figura 3).

Clasificación de principales remanentes de hábitat potencial. La clasificación de las 12 unidades espaciales de hábitat potencial para el tapir en Guatemala se presenta en la Tabla 4 y Figura 4. Las unidades espaciales de mayor importancia son el noreste de la RBM (incluyendo zonas núcleo, de usos múltiples y de amortiguamiento) y las correspondientes a la Reserva de Biosfera Sierra de las Minas y Parque Nacional Sierra del Lacandón.

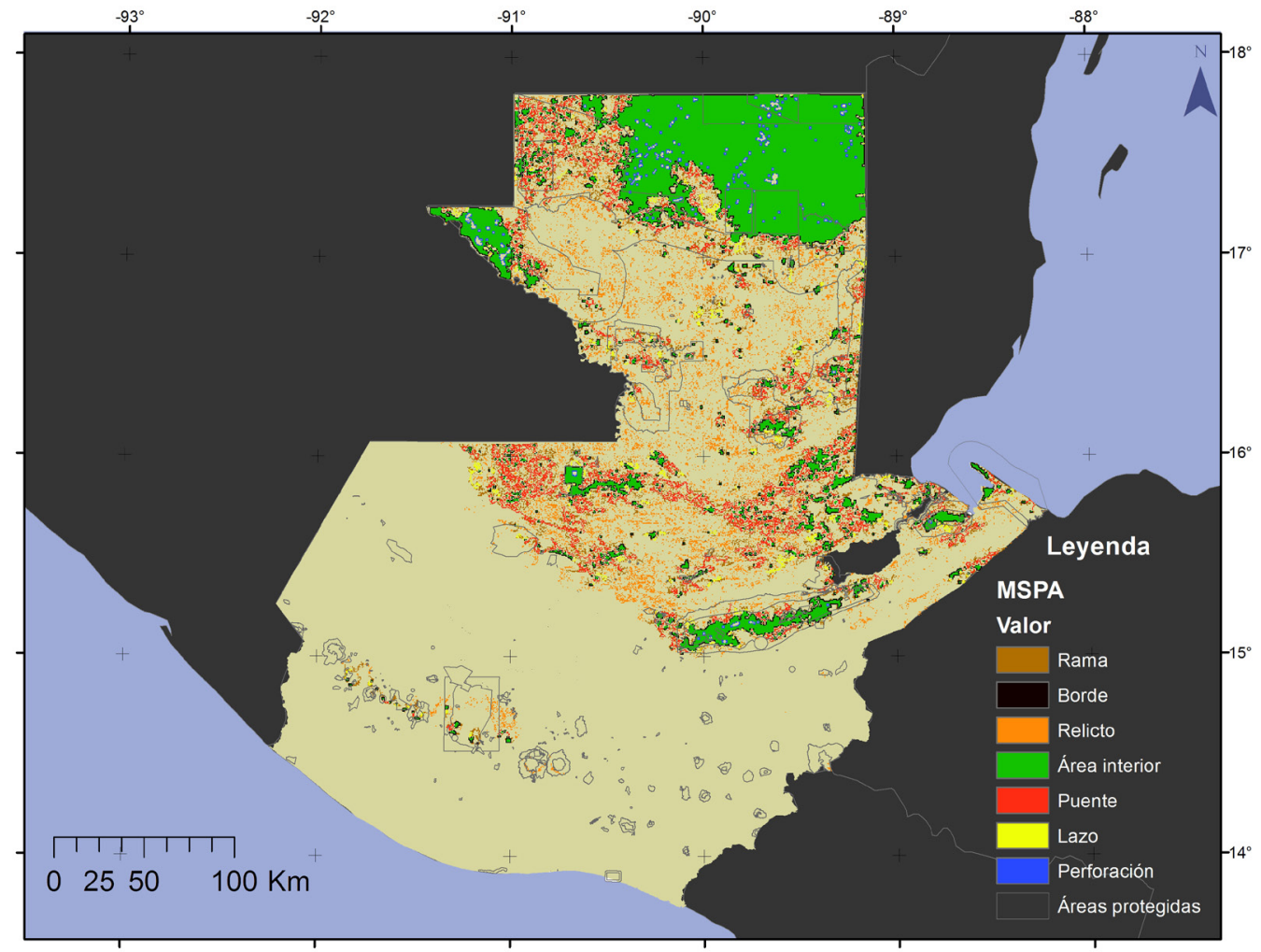

Figura 2. Mapa de las siete clasificaciones del análisis morfológico de los patrones espaciales del hábitat potencial de Tapirus bairdii en Guatemala. 
Tabla 1. Clasificaciones del análisis morfológico de los patrones espaciales del hábitat potencial de Tapirus bairdii en Guatemala

\begin{tabular}{lcc}
\hline \multicolumn{1}{c}{ Clasificación de la cobertura } & $\begin{array}{c}\text { Número de elementos (frecuencia) } \\
\text { que comprende el hábitat }\end{array}$ & Porcentaje del hábitat (\%) \\
\hline Área interior & 639 & 43 \\
Relicto & 4,620 & 14 \\
Perforación & 208 & 2 \\
Borde & 1,712 & 10 \\
Lazo & 341 & 4 \\
Puente & 284 & 17 \\
Rama & 3,775 & 11 \\
Total & 11,579 & 100 \\
\hline
\end{tabular}

\section{Discusión}

Modelado de la distribución potencial. El resultado del modelo de distribución potencial del tapir en Guatemala es semejante al obtenido por Schank et al. 2015. En ambos casos se evidencia alta probabilidad de presencia de la especie en la sección noreste de la RBM, la cual forma parte del bloque transfronterizo de la Selva Maya (Reserva de la Biosfera Calakmul en México, RBM en Guatemala y Río Bravo en Belice), y la sección oeste donde se ubica el Parque Nacional Sierra del Lacandón (colindante con la Reserva de la Biosfera Montes Azules, México). De acuerdo con Naranjo (2009)

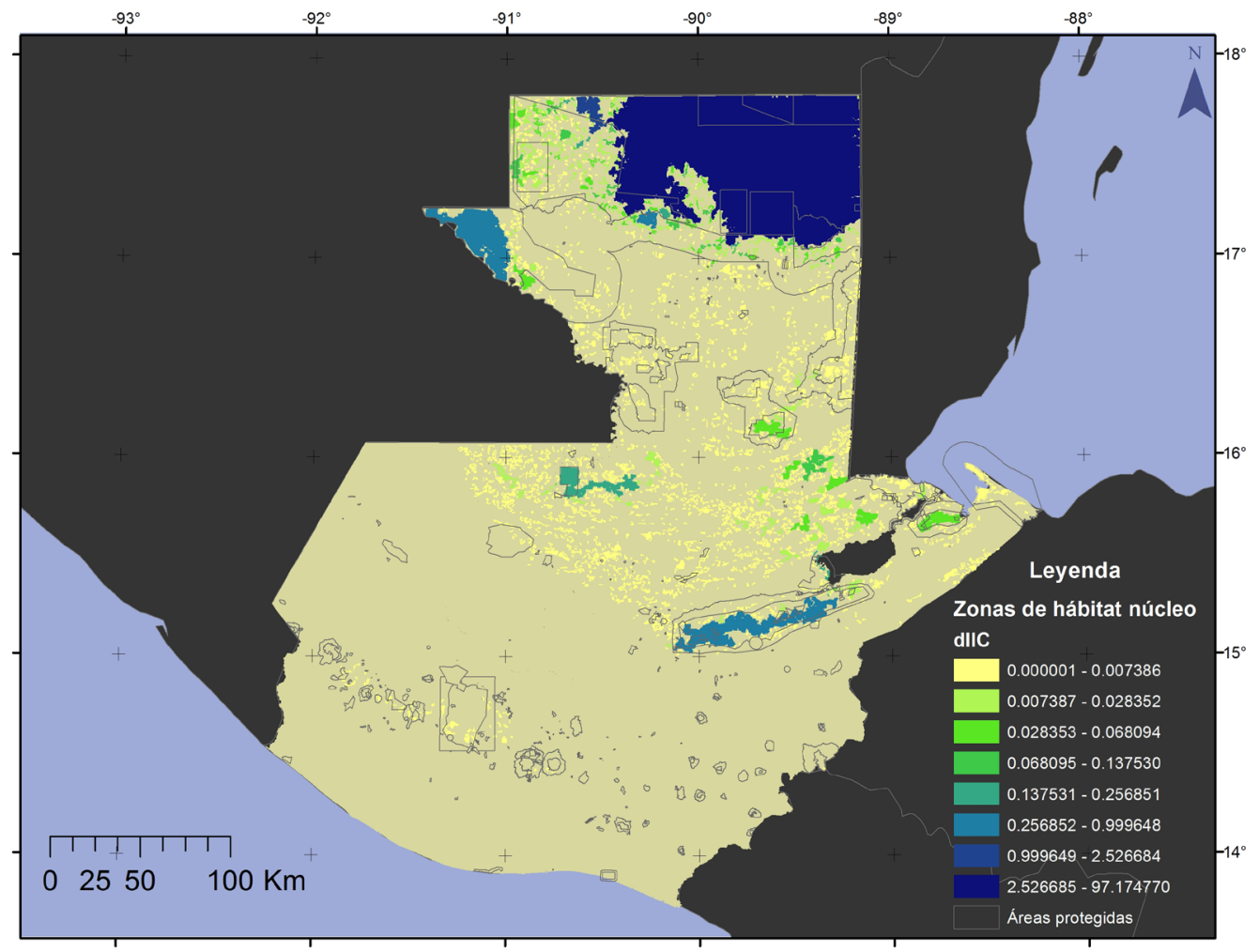

Figura 3. Mapa del Índice Integral de Conectividad para 12 remanentes boscosos de mayor tamaño dentro del área de distribución de Tapirus bairdii en Guatemala. En color amarillo claro se encuentran los remanentes con menor dIIC y en color azul los remanentes con mayor dIIC. 
estas zonas de México son de gran importancia ya que albergan alrededor del $50 \%$ de la población de tapir en ese país.

Esta región de alta probabilidad de distribución incluye en su extremo inferior al Parque Nacional Laguna Lachuá, Reserva de Biosfera Sierra de las Minas y áreas protegidas de Izabal, donde su presencia ha sido reportada durante la última década. Al igual que Schank et al. 2015, el presente modelo sobreestima (aunque en menor escala) la presencia de tapir en la región central y sur del país (específicamente en la cadena volcánica), donde existe alta fragmentación y cambio de uso del suelo debido a las actividades agropecuarias (p.e. algodón, caña de azúcar, ganadería extensiva) que han contribuido a su extinción local, sin embargo, es un área de distribución histórica (Alston 1918; Saunders et al. 1950).

Es importante resaltar que las zonas de alta probabilidad de distribución de la especie en el modelo coinciden en un alto porcentaje con áreas protegidas. De acuerdo al análisis del modelo, las áreas protegidas constituyen los últimos refugios donde se alberguen poblaciones de tapir en el país, aspecto que evidencia la importancia que tienen estos espacios en la supervivencia de esta especie y de las especies que están asociadas a su hábitat.

El alto porcentaje de contribución de la variable BIO17 (precipitación del trimestre más seco) puede sugerir, de manera similar a los resultados de Carrillo-Reyna et al. (2015) para la península de Yucatán, que los tapires no se distribuyen en las zonas más calientes con escasa precipitación. En este sentido la disponibilidad de agua puede ser un factor limitante de la distribución de la especie, especialmente en ambientes con poca disponibilidad de agua superficial como ocurre en la región RBM-NO, al igual que áreas aledañas de México en el estado de Campeche (Pérez-Córtez et al. 2012; Carrillo-Reyna et al. 2015)

Análisis morfológico del patrón espacial. Las zonas interiores constituyen el mayor porcentaje de acuerdo al análisis MSPA, situación que se evidencia principalmente en el gran bloque de la RBM-NE, donde la cobertura forestal es mayor y la fragmentación es baja. La pérdida de hábitat y la fragmentación son las principales amenazas para la especie en la región (Castellanos et al. 2008). Las poblaciones están quedando cada vez más aisladas, lo que las hace más vulnerables ante la cacería y las enfermedades, reduciendo su éxito reproductivo y la supervivencia de los juveniles, entre otros aspectos (Deyoung y Honeycutt 2005). En Brasil, Gonçalves (2007) evaluó el efecto de la fragmentación en cinco especies de mamíferos y encontró que esta las afecta en diferente escala de acuerdo con el tamaño corporal. Sin lugar a duda el patrón morfológico de la cobertura forestal tiene un efecto directo en la supervivencia de las especies (Akçakaya y Brook 2009), por lo que su análisis aporta información esencial para el manejo del hábitat del tapir.

Las categorías de relicto y rama fueron las clasificaciones más numerosas $(4,620$ y 3,775 respectivamente) pero no las más representativas ( $14 \%$ y $11 \%$ respectivamente) obtenidas a partir del Análisis Morfológico de los Patrones Espaciales. Estos dos elementos evidencian el proceso de fragmentación. En el caso de relicto los resultados muestran que existe una mayor área con pequeños fragmentos de hábitat, que son producto de la pérdida paulatina del hábitat; a pesar de constituir zonas potenciales de alimentación es importante recuperar la conexión de éstas hacia áreas interiores, puesto que por sí mismas no pueden albergar poblaciones viables (Correa y Mendoza 2013). Lo anterior se ve reflejado en la zona sur de la RBM, las Verapaces e Izabal, donde la fragmentación del hábitat se ha incrementado durante los últimos 
Tabla 2. Descripción de 12 unidades espaciales de mayor tamaño en el hábitat potencial del Tapirus bairdii en Guatemala.

\begin{tabular}{|c|c|c|c|c|c|}
\hline \multirow{2}{*}{ No. } & \multirow{2}{*}{ Unidad espacial } & \multirow{2}{*}{$\begin{array}{l}\text { Área } \\
\text { total } \\
\left(\mathbf{k m}^{2}\right)\end{array}$} & \multicolumn{2}{|c|}{$\begin{array}{c}\text { Mecanismos formales } \\
\text { de protección (áreas } \\
\text { protegidas) }\end{array}$} & \multirow[t]{2}{*}{ Ubicación } \\
\hline & & & $\begin{array}{l}\text { Extensión } \\
\left(\mathbf{k m}^{2}\right)\end{array}$ & $\begin{array}{l}\text { Porcentaje } \\
\text { (\%) }\end{array}$ & \\
\hline 1 & RBM-NE & $9,546.51$ & $9,536.9$ & 100 & $\begin{array}{l}\text { Bloque ubicado en el Noreste } \\
\text { de la Reserva de Biosfera } \\
\text { Maya. Incluye Zonas núcleo, } \\
\text { Zona de amortiguamiento y } \\
\text { Zona de usos múltiples }\end{array}$ \\
\hline 2 & Sierra de las Minas & 849.2 & 846.9 & 100 & $\begin{array}{l}\text { Reserva de Biosfera Sierra de } \\
\text { las Minas }\end{array}$ \\
\hline 3 & RBM-O & 841.8 & 837.6 & 100 & $\begin{array}{l}\text { Parque Nacional Sierra de } \\
\text { Lacandón }\end{array}$ \\
\hline 4 & Ixcán-Lachuá-Chinajá & 384.9 & 125.3 & 33 & $\begin{array}{l}\text { Complejo: Ecorregión Lachuá } \\
\text { con remanentes en Ixcán y } \\
\text { Chinajá }\end{array}$ \\
\hline 5 & Montañas Chocón Nacional & 209.94 & 0 & 0 & $\begin{array}{l}\text { Complejo de remanentes en } \\
\text { cerros sin protección }\end{array}$ \\
\hline 6 & Sierra Santa Cruz & 145.44 & 0 & 0 & $\begin{array}{l}\text { Área de protección especial } \\
\text { Santa Cruz }\end{array}$ \\
\hline 7 & Cerro San Gil & 134.29 & 134.3 & 100 & $\begin{array}{l}\text { Reserva Protectora de } \\
\text { Manantiales Cerro San Gil }\end{array}$ \\
\hline 8 & Xutilhá & 121.12 & 119.8 & 99 & $\begin{array}{l}\text { Refugio de Vida Silvestre } \\
\text { Xutilhá }\end{array}$ \\
\hline 9 & RBM-NO & 105.75 & 105.7 & 100 & $\begin{array}{l}\text { Complejo de remanentes en } \\
\text { Parque Nacional Laguna del } \\
\text { Tigre y Biotopo Protegido } \\
\text { Laguna del Tigre - Río } \\
\text { Escondido }\end{array}$ \\
\hline 10 & Punta de Manabique & 61.39 & 61.4 & 100 & $\begin{array}{l}\text { Refugio de Vida Silvestre } \\
\text { Punta de Manabique }\end{array}$ \\
\hline 11 & Montañas Mayas & 27.79 & 27.8 & 100 & $\begin{array}{l}\text { Reserva de Biosfera Montañas } \\
\text { Mayas }\end{array}$ \\
\hline 12 & Río Sarstún & 27.21 & 27.2 & 100 & $\begin{array}{l}\text { Área de usos múltiples Río } \\
\text { Sarstún }\end{array}$ \\
\hline
\end{tabular}

años reduciendo la cobertura forestal dentro de las áreas protegidas existentes y aislándolas aún más (GIMBOT 2014).

De acuerdo con Soille y Vogt (2009) las áreas categorizadas como puente contribuyen a conectar físicamente los fragmentos de hábitat, favoreciendo la conectividad entre áreas. Sin embargo, es importante realizar un análisis más detallado, ya que de acuerdo con Correa y Mendoza (2013) estos elementos pueden ser clave para la conectividad entre áreas pero también pueden ser el resultado de la reducción de los fragmentos de hábitat por intervención antrópica.

Conectividad de los remanentes de hábitat potencial. El resultado del análisis de conectividad concuerda con los obtenidos por García et al. (2011), quienes reportan que el dIIC más alto corresponde a la Selva Maya. Además de la integridad ecológica de la RBM, su colindancia con otras zonas boscosas de México y Belice (Mendoza 
et al. 2013; Carrillo-Reyna et al. 2015; Schank et al. 2015) hace de ésta la zona más importante por la conectividad que presenta tanto a nivel interno como externo, contribuyendo de esta forma a mantener el flujo de las poblaciones de fauna silvestre como las de tapir.

Los remanentes boscosos que le siguen en orden de importancia de acuerdo al dIIC son el oeste de la RBM, representado por Sierra del Lacandón, los bloques de Sierra de las Minas y la Ecoregión Lachuá. Estas áreas constituyen los últimos remanentes de mayor tamaño, donde a pesar de no tener conexión con otras áreas, a excepción de Lacandón que si presenta conexión externa con la Reserva de la Biosfera Montes Azules, la conectividad interna aún es relativamente alta.

Al igual que lo reportado por García et al. (2011), el resto de áreas ubicadas en Izabal y la región sur de Petén presentaron un dIIC bajo, evidenciando la baja conectividad con otras áreas circundantes, así como la baja conectividad que existe a nivel interno, resultado de la fragmentación de estos bloques. No debe descartarse que estas zonas puedan ser utilizadas como remanentes de paso (stepping stones) y como núcleos de restauración.

Muench (2006) Ilevó a cabo una caracterización del estado de la conectividad en tres zonas de la selva Lacandona en México e identificó 40 corredores, clasificados en seis categorías y localizados sobre seis unidades del paisaje. Los resultados de la conectividad de los remanentes boscosos en conjunto con la información de los patrones morfológicos obtenidos en este estudio pueden ayudar a identificar a mayor detalle las áreas donde la conectividad externa puede ser mejorada a través de diferentes mecanismos de manejo como el establecimiento de programas de restauración ecológica.

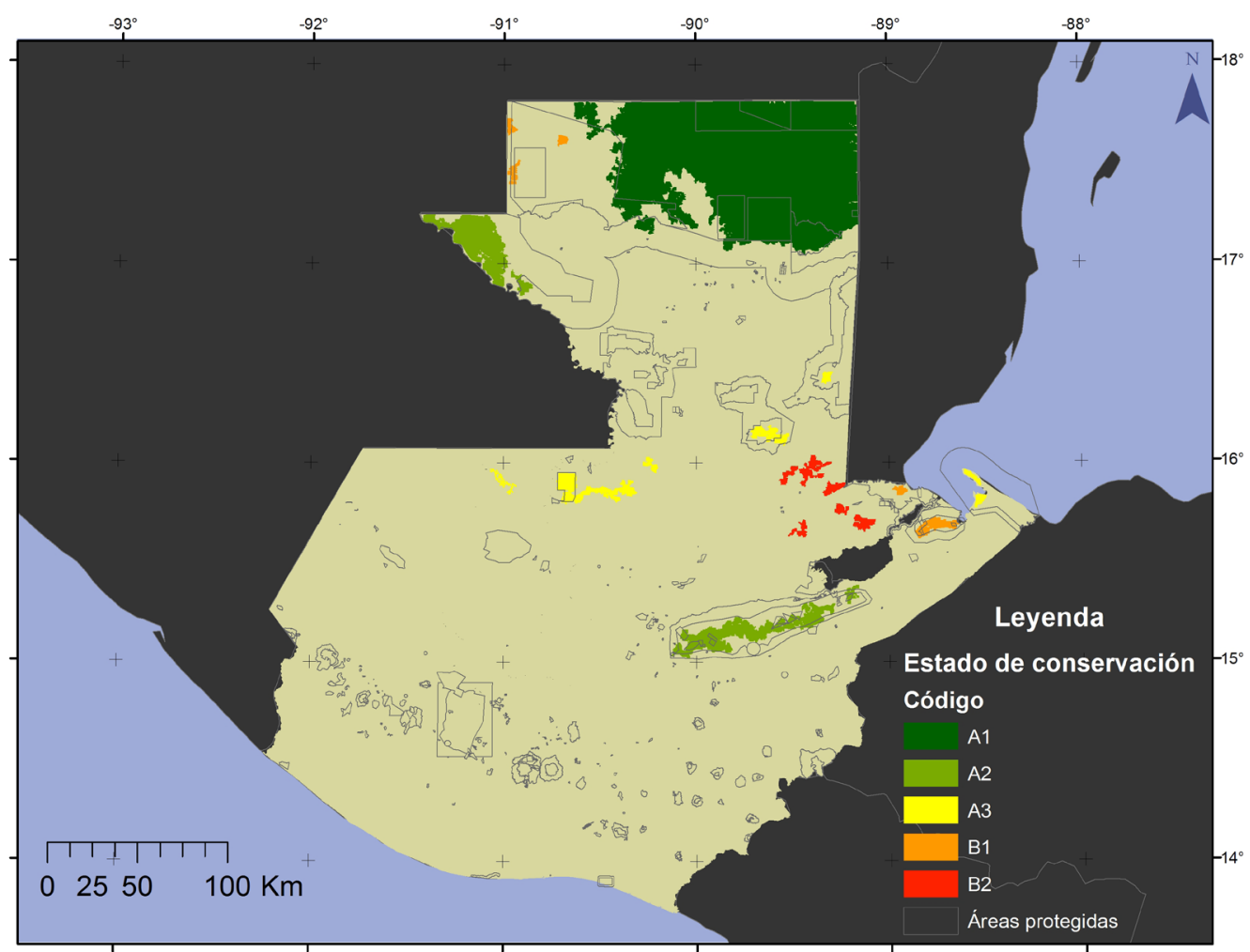

Figura 4. Mapa de clasificación de 12 unidades espaciales de mayor dentro hábitat potencial de Tapirus bairdii en Guatemala. 
Clasificación de principales remanentes de hábitat potencial. El remanente que corresponde al noreste de la RBM ya ha sido identificado como el área de mayor importancia para la conservación del tapir (Matola et al. 1997; García et al. 2009; 2010b; Schank et al. 2015). Naranjo (2009) identificó como una zona de importancia global para las poblaciones de tapir al remanente de Calakmul, que colinda con este bloque de la RBM, por contar con la mayor población de tapir en vida silvestre a nivel global.

Con respecto a los remanentes tipo A2, Sierra de las Minas es un área con baja conectividad hacia otros remanentes de hábitat pero con alta conectividad interna debido a su extensión, mientras que Sierra del Lacandón presenta alta conectividad tanto interna por su extensión como hacia otros remanentes, ya que colinda con la Reserva de la Biosfera Montes Azules en la Selva Lacandona (Muench 2006; Naranjo 2009).

Tabla 3. Índice Integral de Conectividad de las 12 unidades espaciales de mayor tamaño dentro del hábitat potencial de del Tapirus bairdii en Guatemala.

\begin{tabular}{llr}
\hline & \multicolumn{1}{c}{ Unidades espaciales } & dIIC promedio \\
\hline $\mathbf{1}$ & RBM-NE & 33.50566200 \\
$\mathbf{2}$ & Sierra de las Minas & 0.51311405 \\
$\mathbf{3}$ & RBM-O & 0.38876015 \\
$\mathbf{4}$ & Ixcán-Lachuá-Chinajá & 0.09761087 \\
$\mathbf{5}$ & Montañas Chocón Nacional & 0.05743970 \\
$\mathbf{6}$ & Sierra Santa Cruz & 0.03534297 \\
$\mathbf{7}$ & Cerro San Gil & 0.03396800 \\
$\mathbf{8}$ & Xutilhá & 0.04563750 \\
$\mathbf{9}$ & RBM-NO & 0.08055553 \\
$\mathbf{1 0}$ & Punta de Manabique & 0.00195510 \\
$\mathbf{1 1}$ & Montañas Mayas & 0.00638260 \\
$\mathbf{1 2}$ & Río Sarstún & 0.00428770 \\
\hline
\end{tabular}

De acuerdo a los resultados obtenidos en la clasificación de los principales remanentes de hábitat potencial y a los análisis realizados, se puede evidenciar una disminución y deterioro del hábitat del tapir en el país en los últimos cinco años, en relación con evaluaciones previas realizadas para el Sistema Guatemalteco de Áreas Protegidas (SIGAP) por García et al. (2008b, 2009, 2010b). Algunos de los fragmentos que fueron considerados previamente han desaparecido o se ha reducido el área boscosa a fragmentos menores a $1 \mathrm{~km}^{2}$, como es el caso del remanente correspondiente a la Reserva Biológica San Román, en la cual García et al. (2009) obtuvieron en el año 2008 un registro de presencia de la especie, y para el 2012 la cobertura boscosa había sido reemplazada casi en su totalidad por monocultivo de palma africana (GIMBOT 2014). Incluso en la RBM-NE, el remanente de mayor extensión en el país, se una reducción de la cobertura y modificación del patrón morfológico espacial. Lo anterior, permite deducir que el estado de conservación de las poblaciones silvestres de la especie en el país es vulnerable, ya que existe una tendencia continua a la pérdida y degradación de hábitat con la consecuente reducción o extinción de poblaciones locales de tapires. Se hace evidente la necesidad del desarrollo de herramientas de 
gestión del territorio e implementación de acciones que reviertan esta tendencia así como el fortalecimiento del SIGAP.

\section{Agradecimientos}

El presente estudio se realizó gracias al apoyo financiero de: Fondo Nacional para el Desarrollo de la Ciencia y la Tecnología (FODECYT) otorgado por el Consejo Nacional de Ciencia y Tecnología (CONCYT) y la Secretaría Nacional de Ciencia y Tecnología (SENACYT), Fondo Nacional para la Conservación de la Naturaleza (FONACON), Dirección General de Investigación (DIGI) de la Universidad de San Carlos de Guatemala (USAC), Fondo para la Conservación del Tapir del Grupo de Especialistas del Tapir (TSG) de la UICN y Jacksonville Zoo \& Gardens, y el apoyo administrativo de la Organización Nacional para la Conservación y el Ambiente (ONCA). Agradecemos también el apoyo del Centro de Datos para la Conservación (CDC) del Centro de Estudios Conservacionistas (CECON) y las instituciones por compartir información de interés: Consejo Nacional de Áreas Protegidas (CONAP) (Subregionales Petén e Izabal), Instituto de Antropología e Historia Natural (IDAEH),

Tabla 4. Clasificación de 12 unidades espaciales de mayor tamaño dentro del potencial de Tapirus bairdii en Guatemala.

\begin{tabular}{cccccc}
\hline No. & Remanente & Presencia & Conectividad & Protección & Código \\
\hline 1 & RBM-NE & Sí & Alta & Alta & A1 \\
2 & Sierra de las Minas & Sí & Media & Alta & A2 \\
3 & RBM-O & Sí & Media & Alta & A2 \\
4 & Montañas Mayas & Sí & Baja & Alta & A3 \\
5 & Punta de Manabique & Sí & Baja & Alta & A3 \\
6 & Xutilhá & Sí & Baja & Alta & A3 \\
7 & Ixcán-Lachuá-Chinajá & Sí & Baja & Media & A3 \\
8 & Cerro San Gil & No & Baja & Alta & B1 \\
9 & Río Sartstún & No & Baja & Alta & B1 \\
10 & RBM-NO & No & Baja & Alta & B1 \\
11 & Sierra Santa Cruz & No & Baja & Nula & B2 \\
12 & Montañas Chocón Nacional & No & Baja & Nula & B2 \\
\hline
\end{tabular}

Fundación Defensores de la Naturaleza (FDN), Fundación para el Ecodesarrollo y la Conservación (FUNDAECO), AprovaSank, Instituto Nacional de Bosques (INAB), Fundación PROPETEN, Fundación para la Conservación del Ambiente "Mario Dary Rivera" (FUNDARY), WildlifeConservationSociety (WCS), Parque Nacional Zoológico La Aurora y la Editorial Universitaria de la Universidad de San Carlos de Guatemala. Por último, agradecemos la participación de I. Gómez, L. García, F. Castillo, G. Rodríguez y E. Alburez.

\section{Literatura citada}

Акс̧акаYA, R., y B. BrooK. 2009. Methods for determining viability of wildlife populations in large landscapes. Pp 688 en Models for planning wildlife conservation in large landscapes. (Millspaugh, J., y F. Thompson, eds.). 
Alston, E. 1918. Mammalia (1879-1882). En Biologia Central-Americana (Goodman, F. y O. Salvin, eds.). Bernard Quaritch Ltd. London, UK.

Baur, E. 1999. Estudio de la cacería de subsistencia en la Concesión Forestal de Carmelita, San Andrés, Petén. Informe interno. Conservation International - ProPetén. Guatemala.

Bestelmeyer, B., y L. Alonso. 2000. Evaluación biológica de los sistemas acuáticos del Parque Nacional Laguna del Tigre, Petén, Guatemala. RAP Bulletin of Biological Assessment. Conservación Internacional.

Botello, F., J. Sánchez-Hernández, O. Hernández, D. Reyes-Chávez, y V. Sánchez-Cordero. 2014. Registros notables del tapir centroamericano (Tapirus bairdii) en la Sierra Mixe, Oaxaca, México. Revista Mexicana de Biodiversidad 85:995-999.

Carrillo-Reyna, N., R. Reyna-Hurtado, y B. Schmook. 2015. Abundancia relativa y selección de hábitat de Tapirus bairdii en las reservas de Calakmul y Balam Kú, Campeche, México. Revista Mexicana de Biodiversidad 85:202-207.

Carrillo, N., H. Weissenberger, y R. Reyna-Hurtado. 2015. Distribución potencial del Tapir Centroamericano en la península de Yucatán. Therya 6:575-596.

Castellanos, A., C. Foerester, D. J. lizcano, E. Naranjo, E. Cruz-Aldan, I. Lira-Torres, R. Samudio, S. Matola, J. Schipper, y J. Gonzalez-Maya. 2008. Tapirus bairdii. The IUCN Red List of Threatened Species 2008: e.T21471A9284327. http://dx.doi.org/10.2305/IUCN. UK.2008.RLTS.T21471A9284327.en. Downloaded on 12 October 2015.

Consejo Nacional de Áreas Protegidas (CONAP). 2015. Listado de Áreas Protegidas, Sistema Guatemalteco de Áreas Protegidas. Departamento de Unidades de Conservación. Guatemala. Disponible en: file:///C:/Users/Investigacion/Downloads/201501listados igappblico\%20(1).pdf

Correa, C., y M. Mendoza. 2013. Análisis morfológico de los patrones espaciales: una aplicación en el estudio multitemporal (1975-2008) de los fragmentos de hábitat de la cuenca del Lago Cuitzeo. Michoacán, México. GeoSIG 5:50-63.

Deyoung, R., y R. Honeycutt. 2005. The molecular toolbox: genetic techniques in wildlife ecology and management. Journal of Wildlife Management 69:1362-1384.

García, M. 2006. Caracterización de la dieta y el hábitat del tapir (Tapirus bairdii Gill, 1865) en ecosistemas ribereños del Parque Nacional Laguna Lachuá, Cobán, Alta Verapaz, Guatemala. Tesis de Licenciatura en Biología, Facultad de Ciencias Químicas y Farmacia, Universidad de San Carlos de Guatemala.

García, M. J., Castillo, F. y R. Leonardo. 2011. Evaluación preliminar de la conectividad de hábitat para el tapir centroamericano (Tapirus bairdii) en Guatemala. The Newsletter of the IUCN /SSC Tapir Specialist Group 20:20-24.

García, M., R. Leonardo, I. Gómez, y L. García. 2008в. Estado actual de conservación del Tapir (Tapirus bairdii) en el Sistema Guatemalteco de Áreas Protegidas. Informe Técnico Final, FODECYT 120-06, Guatemala.

García, M., R. Leonardo, I. Gómez, y L. García. 2008b. Estado actual de conservación del tapir (Tapirus bairdii) en el Sistema Guatemalteco de Áreas Protegidas. Mesoamericana 12:67.

García, M., R. Leonardo, I. Gómez, L. García, y F. Castillo. 2010a. Los Biotopos Universitarios como sitios prioritarios para la conservación del hábitat del tapir (Tapirus bairdii Gill.) en Guatemala. Ciencia y Conservación 1:32-39.

García, M., R. Leonardo, F. Castillo, I. Gómez, y L. García. 2010b. El Tapir centroamericano (Tapirus bairdii) como herramienta para el fortalecimiento del Sistema Guatemalteco de Áreas Protegidas. Informe Final, Dirección General de Investigación, Universidad de San Carlos de Guatemala, Guatemala. 
García, M., R. Leonardo, L. García, e I. Gómez. 2009. Estado actual de conservación del Tapir (Tapirus bairdii) en el Sistema Guatemalteco de Áreas Protegidas. Informe Final, FONACON, Guatemala.

García, R., y J. Radachowsky (Eds). 2004. Evaluación ecológica rápida del Parque Nacional Mirador Río Azul, Petén, Guatemala. Informe interno, Wildlife Conservation Society, Programa para Guatemala. Guatemala.

García, R. et AL. 2005. Los jaguares del corazón del Parque Nacional Tikal, Petén, Guatemala. Informe interno, Wildlife Conservation Society, Programa para Guatemala, Guatemala.

Gómez, A. 2011. Comparación de la diversidad y abundancia de mamíferos en dos localidades de la Reserva de la Biosfera Visis-Cabá, Guatemala. Tesis de Licenciatura en Biología. Facultad de Ciencias Químicas y Farmacia, Universidad de San Carlos de Guatemala.

Gonçalves, A. 2007. Causes of spatial genetic structure in mammals: a case study in the Atlantic forest, Brazil. Tesis para optar al grado de doctor en filósofia. University of British Columbia, Canadá.

Grupo Interinstitucional de Monitoreo de Bosques y Uso de la Tierra [GiMBOT]. 2014. Mapa de bosques y uso de la tierra 2012 y Mapa de cambios en uso de la tierra 2001- 2010 para estimación de emisiones de gases de efecto invernadero. Informe técnico, GIMBOT. Guatemala.

Hijmans, R. J., S. E. Cameron, J. L. Parra, P. G. Jones, y A. Jarvis. 2005. Very high resolution interpolated climate surfaces for global land areas. International Journal of Climatology 25:1965-1978.

IBARRA, J. 1959. Apuntes de Historia Natural y Mamíferos de Guatemala. Editorial José Pineda de Ibarra. Guatemala.

KawanishI, K. 1995. Camera Monitoring of Human Impacts on Rain Forest Wildlife in Tikal National Park, Guatemala. Tesis de Maestría. Frostburg State University. EE.UU.

March, I. 1994. Situación actual del tapir en México. Serie Monográfica No.1. Chiapas, México: Centro de Investigaciones Ecológicas del sureste.

Matola, S., A. Cuarón, y H. Rubio-Torgler. 1997. Status and action plan of the Baird's Tapir (Tapirus bairdii). En: Tapirs, status survey and conservation action plan (Brooks, D., R. Bodmer y S. Matola, eds). IUCN.

MeDICI, E. P. 2010. Assessing the viability of lowland tapir populations in a fragmented landscape. Tesis de doctorado. University of Kent.

Mendoza, E., T. L. Fuller, , H. A. Thomassen, W. Buermann, D. Ramírez-Mejía, y T. B. Smith. 2013. An assessment of the effectiveness of the Mesoamerican Biological Corridor for protecting potential Baird's tapir (Tapirus bairdii) habitat in southern Mexico. Integrative Zoology 8:34-46.

Morales, J., A. J. Cóbar, F. Ramírez, A. Morales, J. Moreira, R. Leonardo, y M. González. 2005. Programa piloto de conservación y manejo de especies cinegéticas en tres comunidades de la Reserva de Biosfera Sierra de las Minas (RBSM). Proyecto FONACON F12/2004 y Proyecto FODECYT 12-03. Guatemala.

Moreira, J. et al. 2007. The comparative abundance of jaguars in La Gloria-El Lechugal, multiple use zone, Maya biosphere reserve, Guatemala. Informe interno, Wildlife Conservation Society, Programa para Guatemala, Guatemala.

Muench, C. 2006. Corredores de vegetación y conectividad de hábitat para el tapir (Tapirus bairdii) en la Selva Lacandona, Chiapas. Tesis para optar al grado de maestro en ciencias biológicas. Universidad Autónoma de México. UNAM.

Naranjo, E. 2001. El Tapir en México. Biodiversitas 21:9-11.

Naranjo, E. 2009. Ecology and conservation of Baird's tapir in Mexico. Tropical Conservation Science 2:140-158. 
Naranjo, E., y C.VAughan. 2000. Ampliación del Ámbito Altitudinal del Tapir Centroamericano (Tapirus bairdii). Revista de Biología Tropical 48:724.

Novack, A. 2003. Impacts of subsistence hunting on the foraging ecology of jaguar and puma in the Maya Biosphere Reserve. Tesis de maestría. University of Florida. EE.UU.

Pascual-Hortal, L., y S. Saura. 2006. Comparison and development of new graph-based landscape connectivity indices: towards the priorization of habitat patches and corridors for conservation. Landscape Ecology 21:959-967.

Philulps, S. J., y M. Dudik. 2008. Modeling of species distributions with Maxent: new extensions and a comprehensive evaluation. Ecography 31:161-175.

Ruíz, J., y G. Rodríguez. 2006. Abundancia poblacional, movimientos y uso de hábitat del Tapir (Tapirus bairdii), en el Parque Nacional Laguna Lachua, Alta Verapaz, Guatemala (Segunda Fase). Guatemala: Dirección General de Investigación -DIGI-, Universidad de San Carlos de Guatemala.

Ruíz, J., y M. García. 2005. Abundancia poblacional, movimientos y uso de hábitat del Tapir (Tapirus bairdii), en el Parque Nacional Laguna Lachua, Alta Verapaz, Guatemala (Primera Fase). Guatemala: Dirección General de Investigación -DIGI-, Universidad de San Carlos de Guatemala.

Saunders, G. B. 1950. The Game Birds and Shorebirds of Guatemala. Special Scientific Report 5- Wildlife 5:3-98.

Saura, S., y L. Pascual-Hortal. 2007. A new habitat availability index to integrate connectivity in landscape conservation planning: comparison with existing indices and applications to a case study. Landscape and urban planning, DOI 10.1016/j. landurbplan.2007.03.005.

Schank, C., E. Mendoza, V. García, M. V. Cove, C. A. Jordan, G. O'Farrill, N. Meyer, D. J. Lizcano, N. Estrada, C. Poot, y R. Leonardo. 2015. Integrating current range-wide occurrence data with species distribution models to map the potential distribution of Baird's Tapir. The Newsletter of the IUCN /SSC Tapir Specialist Group 24:15-30.

Sollte, P., y P. Vogt. 2009. Morphological segmentation of binary patterns. Pattern recognition letters 30:456-459.

Vogt, P., K. H. Riltters, C. Estreguil, J. Kozak, T. G. Wade, y J. D. Wickham. 2007. Mapping spatial patterns with morphological image processing. Landscape Ecology 22:171177.

Xımenez, F. 1967. Historia Natural del Reino de Guatemala. Editorial José de Pineda Ibarra. Guatemala.

ZaPATA, G., y J. Dyer. 2003. Diseño de una red de áreas protegidas en la amazonía nororiental ecuatoriana: uso combinado de los sistemas de información geográfica y los análisis de viabilidad poblacionales. Lyonia 5:169-178.

Submitted: October 19, 2015

Reviewed: November 30, 2015

Accepted: January 16, 2016

Associated editor: Rafael Reyna 
122 THERYA Vol.7 (1): 107-121 